\title{
Editorial
}

\section{Mathematical Methods and Models in the Natural to the Life Sciences}

\author{
Rehana Naz, ${ }^{1}$ Igor Leite Freire, ${ }^{2}$ Imran Naeem, ${ }^{3}$ and Mariano Torrisi ${ }^{4}$ \\ ${ }^{1}$ Centre for Mathematics and Statistical Sciences, Lahore School of Economics, Lahore 53200, Pakistan \\ ${ }^{2}$ Centro de Matemática, Computação e Cognição, Universidade Federal do ABC, Rua Santa Adélia 166, Bairro Bangu, \\ 09.210-170 Santo André, SP, Brazil \\ ${ }^{3}$ Department of Mathematics, School of Science and Engineering, Lahore University of Management Sciences, Lahore Cantt, \\ Lahore 54792, Pakistan \\ ${ }^{4}$ Dipartimento di Matematica ed Informatica, Università di Catania Viale A. Doria 6, 95125 Catania, Italy
}

Correspondence should be addressed to Rehana Naz; drrehana@lahoreschool.edu.pk

Received 25 August 2014; Accepted 25 August 2014; Published 11 September 2014

Copyright (C) 2014 Rehana Naz et al. This is an open access article distributed under the Creative Commons Attribution License, which permits unrestricted use, distribution, and reproduction in any medium, provided the original work is properly cited.

The search for mathematical models and their analysis was born as a powerful tool for the investigations of nature and as an aid to solve the daily problems that life is offering us. On the other hand, the last decades have witnessed an increased interest in the growth and progress of the study of mathematical methods that are crucial in the quick advancement of the natural, physical, engineering, social, and life sciences.

The purpose of this special issue is to bring together papers covering a wide range of scientific interests concerned with differential equations emanating from mathematical models of classical fields, such as solids and fluid mechanics, or from some fields as biology, economics and finance, and sciences of life that, only in the second part of the last century, have begun to take into consideration the tools offered from mathematics. The mathematical techniques are not only devoted to the search for numerical and exact solutions of the underlying differential equations for general and special problems; they also include new theoretical developments suitable for novel applications of solving real-world problems.

The response to this special issue was beyond our expectation. We received 60 papers to be considered for publication. Those submissions, from different countries and continents, fall in different areas of the above-mentioned research fields. All papers submitted to this special issue went through a rigorous peer-refereeing process. Based on the reviewers reports, thirty-three original research articles have finally been accepted for publication. The contents embrace different qualitative and quantitative techniques, Lie symmetry techniques, numerical methods, stability analysis, and statistical methods to analyze different aspects that are concerned with differential equations arising in economics, finance, biology, physics, and fluid dynamics. It is certainly impossible to provide in this short editorial a more comprehensive description for all articles of this special issue. However, the guest editors believe that the results included reflect some recent trends in research and outline new ideas for future studies. In the following, we briefly describe the significance of the key contributions to our special session.

Lie symmetry methods are powerful tools to find conservation laws/first integrals, reductions, and exact solutions of differential equations. Some papers in our special issue have used Lie symmetry methods to study interesting problems like heat transfer in extended surfaces of different geometries, nonlinear Jaulent-Miodek equation, neutron transport equations in nonhomogeneous media, nonlinear fin equation, and general bond-option pricing equation of mathematical finance. Moreover, different approaches to construct first integrals for ordinary differential equations are compared, as well as symmetries, and their associated first integrals and double reduction of difference equations are also investigated.

Several authors study some interesting fluid mechanics models. Similarity solutions of MHD heat and mass transfer flow of the steady viscous incompressible fluid over a flat 
plate are derived and the ordinary differential equations obtained from similarity transformations are solved by a finite difference scheme known as the Keller Box method. The homotopy analysis method is employed to study the mixed convective heat transfer in an incompressible steady two-dimensional viscoelastic fluid flow over a wedge in the presence of buoyancy effects.

Stability analysis plays a vital role in understanding of the dynamic structure of complex dynamical systems. In our special issue several papers focus on stability analysis of biological systems and these models include Lotka-Voltera predator-prey system with time-delayed feedback, a nutrientphytoplankton model with delay effect, a model of emerging infectious disease leading to amphibian decline, a nutrientalgae-zooplankton system with sinking of algae, a reactiondiffusion system modeling cancer network, Hodgkin-Huxley nonlinear model with constraint, malaria epidemic in Karonga district, stochastic cooperative predator-prey system with Beddington-DeAngelis functional response, a reactiondiffusion phytoplankton-zooplankton system with a double Allee effect on prey, and a stochastic delayed competitive system with impulsive toxicant input in a polluted environment. Dynamics of the oxygen, carbon dioxide, and water interaction across the insect is investigated. A traveling wave analysis is carried out for a tumour-immune interaction model with immunotherapy. Numerical simulations are also performed for few of these papers.

Papers collected in this special issue are also concerned with statistical methods which are important to study some tools essential for financial analysts. These papers include analysis of corporate bond valuation under an infinite dimensional compound Poisson framework, the pricing problem for convertible bonds with higher loan rate, convertible bonds via backward stochastic differential equations, the forward credit default swap with general random loss, and study of financial time series model by copula analysis.

Our special issue contains few papers in which different numerical techniques are employed. Haar wavelet method is used to find numerical solution of the system of Fredholm integral equations and the system of Volterra integral equations. The solution of a class of Lane-Emden equations is derived by the reproducing kernel method. Two nonstandard finite difference schemes to solve the regularized long wave equation are reported in our special issue. A new approach based on the effective combination of Lie symmetry method, homotopy perturbation method, finite element method, and simulation based error reduction techniques is presented and employed to transient nonlinear heat conduction problems. Numerical reduced variable optimization methods via implicit functional dependence are proposed and applied to nontrivail real-world problem. A method is developed for the approximate computation of the frequency-dependent magnetic and electric matrix Green's functions in a rectangular parallelepiped with a perfect conducting boundary. Analytic formulation for the sound absorption of a panel absorber under the effects of microperforation, air pumping, and linear and nonlinear vibrations is reported.

We hope the papers published in this special issue will provide a useful guide to a large community of researchers and will give way to development of new innovative theories in the fields of natural, social, and life sciences.

\section{Acknowledgments}

We thank all the authors who contributed to this special issue. Moreover a special gratitude goes to the honorable reviewers for their hard work, time, and efforts to review all the submitted articles.

Rehana Naz

Igor Leite Freire

Imran Naeem

Mariano Torrisi 


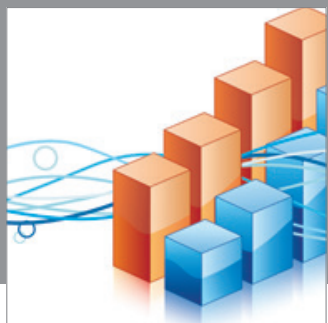

Advances in

Operations Research

mansans

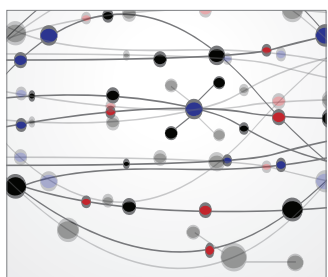

The Scientific World Journal
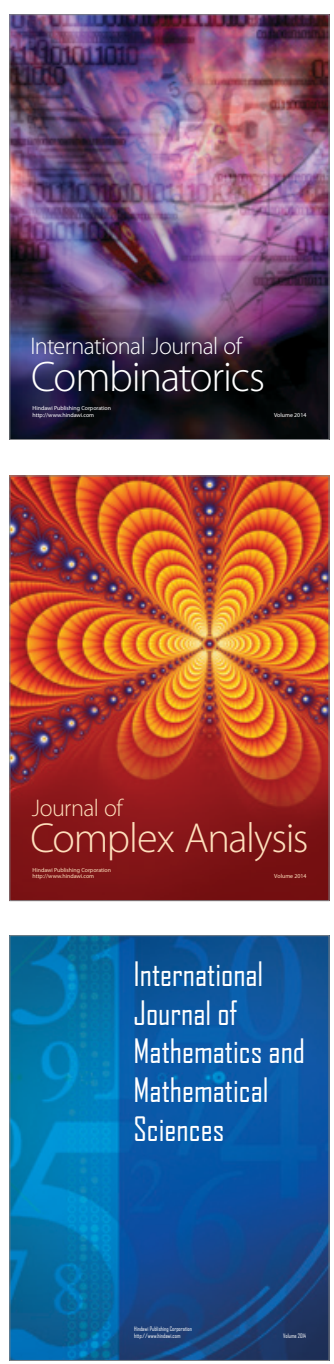
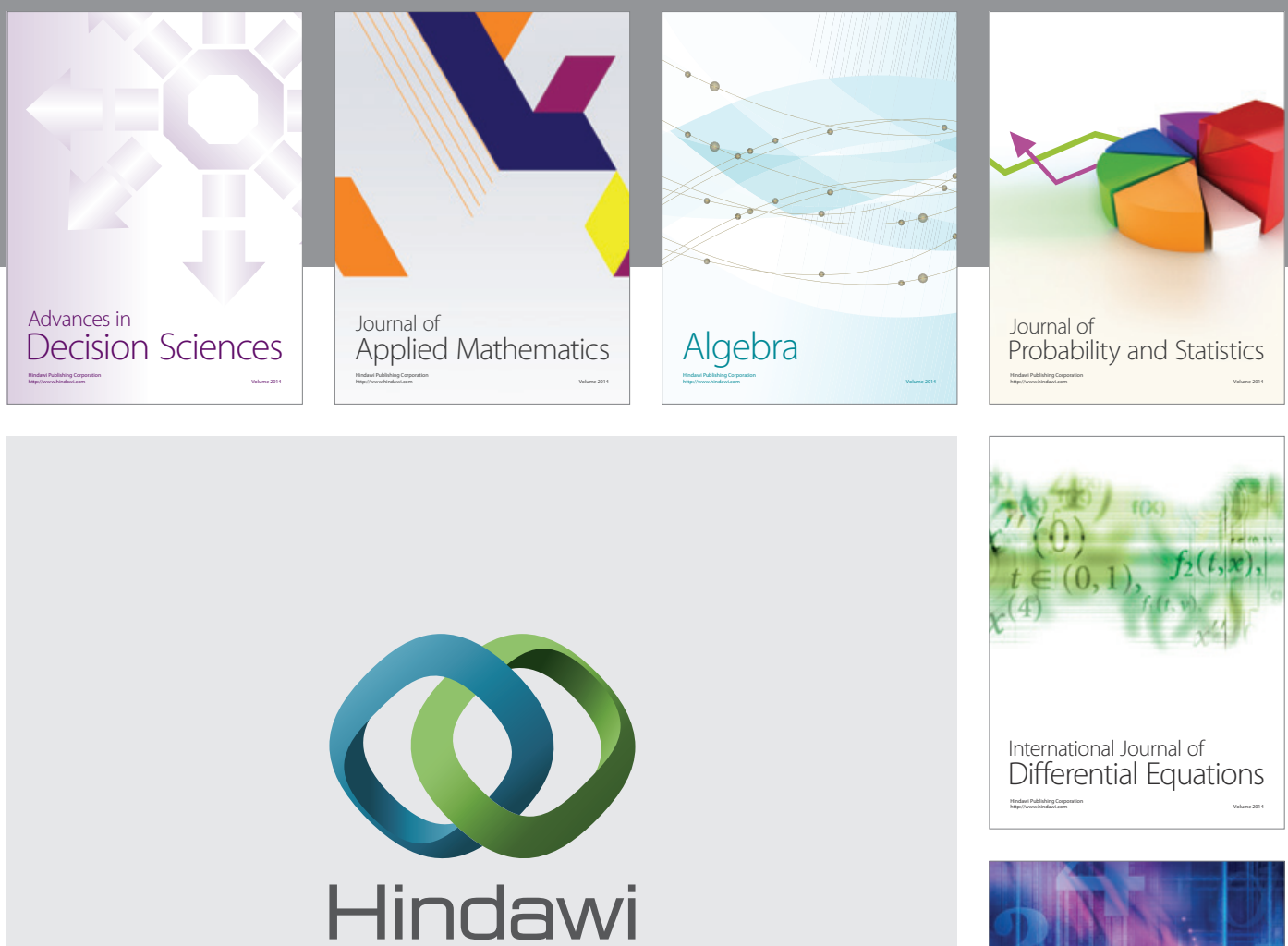

Submit your manuscripts at http://www.hindawi.com
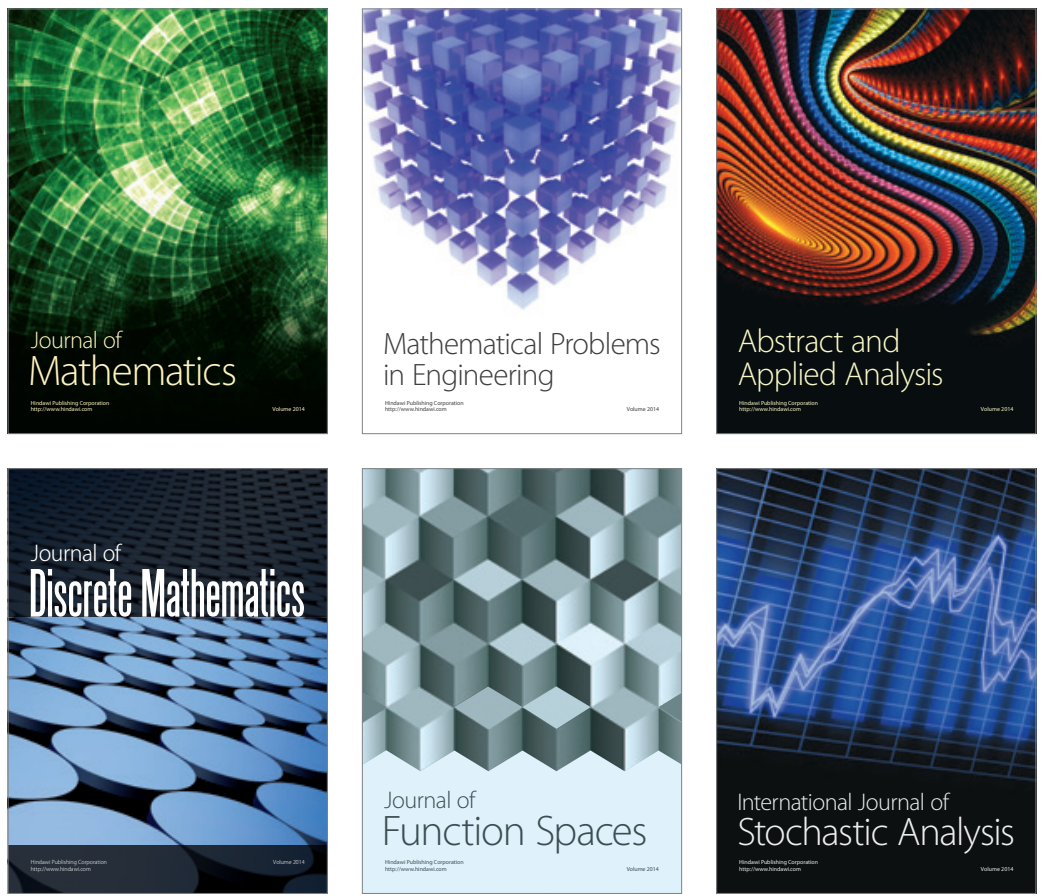

Journal of

Function Spaces

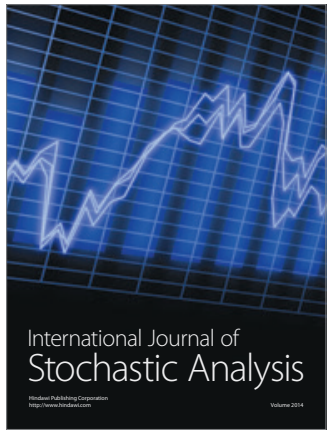

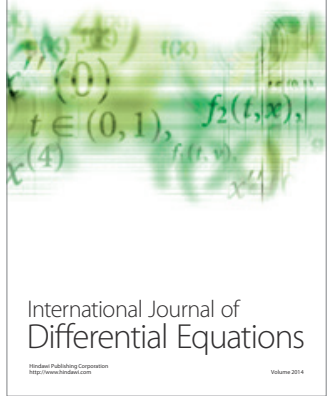
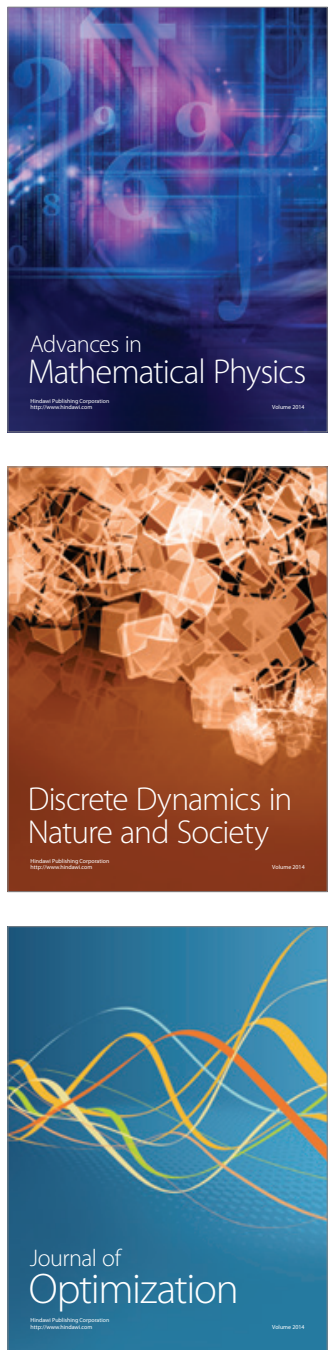